\title{
SINTERIZAÇÃO A PLASMA DO ALUMÍNIO COM A UTILIZAÇÃO A TÉCNICA ACTIVE SCREEN*
}

\author{
Guilherme Santos Garcia ${ }^{1}$ \\ Gilceu dos Santos Cardoso ${ }^{1}$ \\ Lucas Lápis Ely² \\ Sandro Luís Arenhardt ${ }^{3}$ \\ Magnos Marinho ${ }^{3}$ \\ Alexandre da Silva Rocha
}

\section{Resumo}

Este trabalho tem por objetivo avaliar a sinterização a plasma do alumínio com a utilização da técnica Active Screen, em comparação com o processo em forno resistivo. Foi utilizado pó de alumínio atomizado, com granulometria entre 30 e $100 \mu \mathrm{m}$, sendo misturado junto aos pós cerca de $1 \%$ em massa de estearato de zinco como agente lubrificante. Foram confeccionados corpos de prova com $13 \mathrm{~mm}$ de diâmetro e $10 \mathrm{~mm}$ de altura. A compressão dos pós se deu através de uma prensa hidráulica, de ação simples, utilizando pressão de compactação de $600 \mathrm{MPa}$. O tempo te sinterização foi mantido em $60 \mathrm{~min}$ a temperatura de $500^{\circ} \mathrm{C}$, utilizando atmosfera de nitrogênio. Foram realizadas medidas de densificação, antes e após a sinterização, medição de rugosidade e microdureza superficial, bem como análises em MEV da superfície das amostras. O processo a plasma se mostrou satisfatório, sendo capaz de produzir amostras com boa densificação. As medições de microdureza superficial evidenciaram valores semelhantes entre os processos, sendo a amostra sinterizada via plasma a apresentar menor variação de microdureza. Através da análise de EDS das imagens, foi identificada a presença de estearato de zinco em ambos os processos, evidenciado que a extração do lubrificante não foi totalmente eficaz. Ainda, houve uma leve redução da rugosidade no processo a plasma em relação ao convencional.

Palavras-chave: Não ferrosos, gaiola catódica, metalurgia do pó.

\section{PLASMA SINTERING OF ALUMINUM USING THE ACTIVE SCREEN TECHNIQUE}

\section{Abstract}

This study aims to evaluate the plasma sintering of aluminum using the Active Screen technique, compared to the process in resistive furnace. It was used atomized aluminum powder with a particle size between 30 and $100 \mu \mathrm{m}$, being mixed together with about $1 \%$ by weight of zinc stearate as a lubricating agent. Specimens were made with $13 \mathrm{~mm}$ in diameter and $10 \mathrm{~mm}$ in height. The compression of the powder was performed through a single action hydraulic press using 600MPa of compacting pressure. The sintering time was $60 \mathrm{~min}$ at a temperature of $500^{\circ} \mathrm{C}$ using a nitrogen atmosphere. Densification measurements were performed before and after sintering, measurement of roughness and surface microhardness as well as analysis by SEM of the sample surface. This study aims to evaluate the sintering aluminum plasma using the Active Screen technique, compared to the process in resistive furnace. It was used atomized aluminum powder with a particle size between 30 and $100 \mu \mathrm{m}$, after being mixed together with about $1 \%$ by weight of zinc stearate as a lubricating agent. Specimens were made with $13 \mathrm{~mm}$ in diameter and $10 \mathrm{~mm}$ in height. The compression of the post was made through a hydraulic press, single action using $600 \mathrm{MPa}$ compression pressure. The sintering time te was kept $60 \mathrm{~min}$ at a temperature of $500^{\circ} \mathrm{C}$ using a nitrogen atmosphere. densification measurements were performed before and after sintering, measurement of roughness and surface hardness as well as analysis by SEM of the sample surface. The plasma process proved satisfactory, being able to produce samples with good densification. Measurements of microhardness showed similar values between processes, and the sintered sample via plasma shown less variation in microhardness. Through EDS analysis of the images was identified the presence of zinc stearate in both processes, suggesting the extraction of the lubricant was not fully effective. Furthermore, there was a slight decrease in roughness of the plasma process over the conventional process.

Keywords: Non-ferrous, catodic cage, powder metallurgy.

1 Eng. Mecânico, Mestrando Eng. Minas, Metalúrgica e de Materiais, Laboratório de Transformação Mecânica (LdTM), Universidade Federal do Rio Grande do Sul (UFRGS), Porto Alegre, Rio Grande do Sul, Brasil.

2 Graduando, Eng. Metalúrgica, bolsista de Iniciação Científica, LdTM, UFRGS, Porto Alegre, RS, Brasil.

3 Tecnólogo em Fabricação Mecânica, Mestrando Eng. de Minas, Metalúrgica e de Materiais, LdTM, UFRGS, Porto Alegre, RS, Brasil.

4 Engenheiro Mecânico, Doutor em Engenharia de Minas, Metalúrgica e de Materiais, professor adjunto, LdTM, UFRGS, Porto Alegre, RS, Brasil. 


\section{INTRODUÇÃO}

Este trabalho tem como objetivo avaliar a sinterização a plasma do alumínio puro com a utilização da técnica Active Screen como auxílio ao processo.

A utilização da técnica Active Screen, principalmente na nitretação a plasma, é capaz de promover benefícios em relação a nitretação a plasma convencional. Nesta técnica, um dispositivo em formato de gaiola com furos de diâmetro predeterminado, chamada de gaiola catódica, envolve o material a ser tratado. Desta forma, a peça é protegida da ação direta do plasma e a deposição de espécies ativas ocorre através da formação de cátodo oco nos furos da gaiola. A técnica possibilita contornar problemas frequentemente encontrados neste processo, tais como: aquecimento não uniforme, efeito de borda e aquecimento excessivo pela formação de cátodo oco na peça [1].

A sinterização a plasma vem sendo reportada como uma técnica inovadora na metalurgia do pó por aliar o baixo consumo energético à capacidade de processamento em atmosfera controlada a baixa pressão. Ainda, há a possibilidade de tratamentos termoquímicos posteriores no mesmo ciclo térmico, eliminando uma ou mais etapas de processo se realizados por métodos convencionais. Estudos recentes evidenciam a eficácia da sinterização a plasma do alumínio sem o uso de gaiola catódica [2], [3].

Para este trabalho, optou-se por explorar a técnica proposta para a sinterização do alumínio. Este material vem ocupando um espaço importante na indústria automotiva e aeroespacial por aliar baixa densidade e boa resistência mecânica, além de resistência à corrosão. Peças sinterizadas em alumínio apresentam um diferencial nestes segmentos, pois aliam um processo de baixo custo a um material com boas propriedades mecânicas. Ainda, a metalurgia do pó é capaz de produzir peças de forma definida, podendo eliminar processos posteriores de fabricação [4], [5].

Desta forma, este trabalho tem o intuito de explorar a sinterização a plasma do alumínio com a utilização da técnica Active Screen, de modo a verificar sua eficácia em relação à sinterização convencional.

\section{MATERIAIS E MÉTODOS}

Foi utilizado pó de alumínio atomizado (Figura 1), com granulometria entre 30 e $100 \mu \mathrm{m}$, sendo misturado junto ao pó cerca de $1 \%$ em massa de estearato de zinco como agente lubrificante. Foram confeccionados corpos de prova ou amostras verdes com $13 \mathrm{~mm}$ de diâmetro e $10 \mathrm{~mm}$ de altura. A compressão da mistura se deu através de uma prensa hidráulica, de ação simples, utilizando pressão de compactação de 600MPa. 

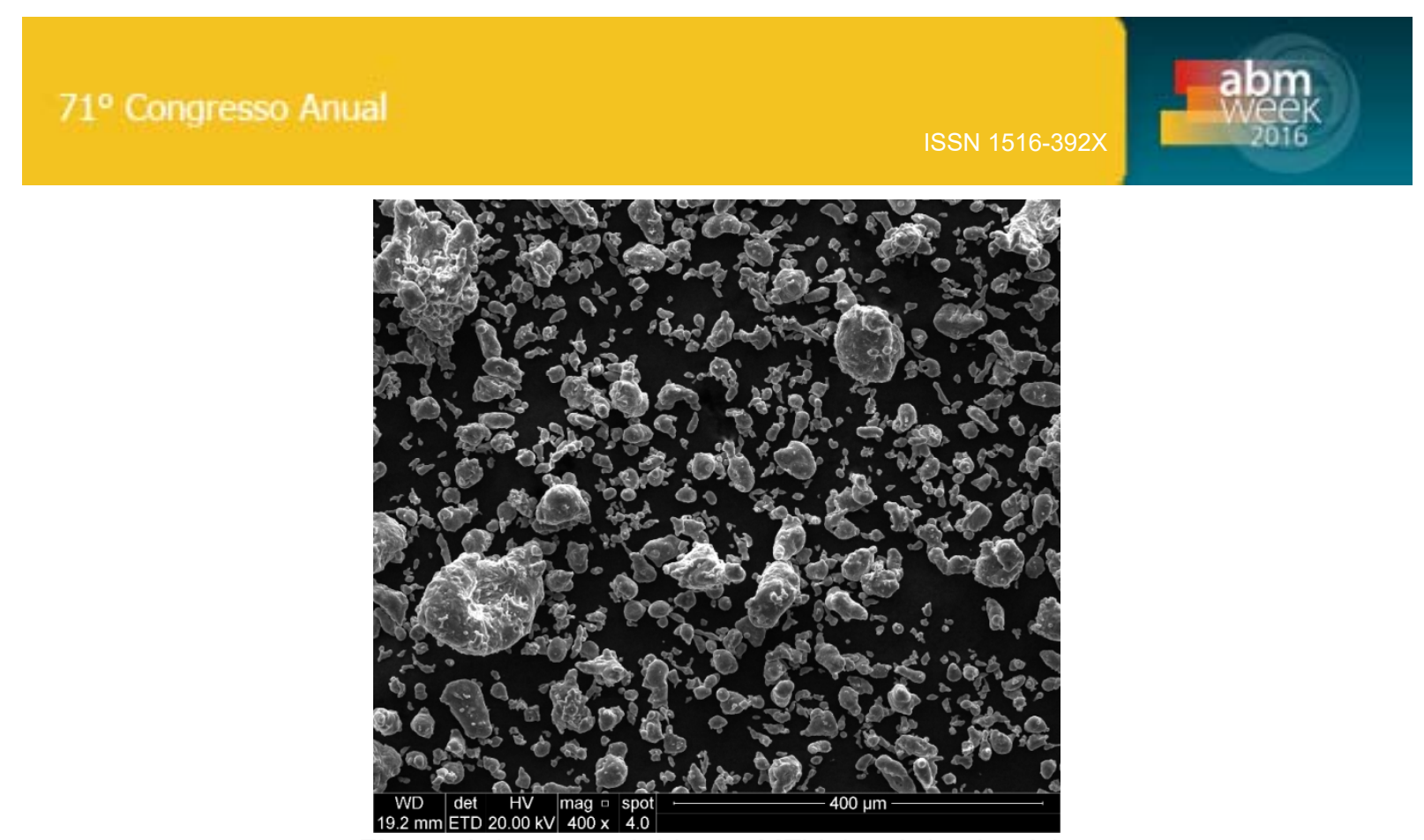

Figura 1. Imagem obtida em MEV do pó de alumínio

Os parâmetros de sinterização podem ser vistos nas Tabelas 1 e 2 . A sinterização convencional foi realizada em um forno tubular com atmosfera de nitrogênio. No processo a plasma, foi utilizada uma câmara de vácuo com pressão de vácuo inicial de $7 \times 10^{-1} \mathrm{mbar}$, sendo utilizados dois gases em etapas diferentes do processo: hidrogênio e nitrogênio. A escolha do hidrogênio se deve pela sua capacidade de, quando ionizado, realizar a limpeza de óxidos da superfície do material. Aliado a isso, foi mantido um patamar de "Limpeza", na sinterização a plasma, para extração do lubrificante.

Tabela 1. Parâmetros da sinterização convencional

\begin{tabular}{lcccc}
\hline Etapa & $\mathbf{T}\left({ }^{\circ} \mathbf{C}\right)$ & $\mathbf{t}(\mathbf{m i n})$ & $\begin{array}{c}\text { Taxa de aquecimento } \\
\left({ }^{\circ} \mathbf{C} / \mathbf{m i n}\right)\end{array}$ & Gás \\
\hline Aquecimento & $25-500$ & 47,5 & 10 & $\mathrm{~N}_{2}$ \\
\hline Sinterização & 500 & 60 & - & $\mathrm{N}_{2}$ \\
\hline
\end{tabular}

Tabela 2. Parâmetros da sinterização a plasma com gaiola catódica

\begin{tabular}{lccccc}
\hline Etapa & $\mathbf{T}\left({ }^{\circ} \mathbf{C}\right)$ & $\mathbf{t}(\mathbf{m i n})$ & $\begin{array}{c}\text { Taxa de } \\
\text { aquecimento } \\
\left({ }^{\circ} \mathbf{C} / \text { min) }\right.\end{array}$ & Gás & $\begin{array}{c}\text { Pressão } \\
\text { (mbar) }\end{array}$ \\
\hline Aquecimento & $25-280$ & 51 & 5 & $\mathrm{H}_{2}$ & $0,5-1,0$ \\
\hline Limpeza & 280 & 90 & - & $\mathrm{H}_{2}$ & 1,0 \\
\hline Aquecimento & $280-500$ & 22 & 10 & $\mathrm{~N}_{2}$ & $1,0-2,5$ \\
\hline Sinterização & 500 & 60 & - & $\mathrm{N}_{2}$ & 2,5 \\
\hline
\end{tabular}

As medidas de densidade foram obtidas através da massa e dimensões dos corpos de prova, quando na condição a verde, e através do método de Arquimedes (que relaciona o volume da peça ao volume de água que a mesma desloca quando submergida em um recipiente com água) para medição de volume quando na condição de sinterizados. Foram calculadas as densificações antes e após a sinterização, cujo cálculo consiste na diferença entre a densidade medida e a densidade teórica do material. Neste caso, adotou-se como densidade teórica a do alumínio puro, de $2,7 \mathrm{~g} / \mathrm{cm}^{3}$.

Foram realizadas medições de microdureza na superfície das amostras, com cargas de 50 e 500g, utilizando um microdurômetro modelo ISH-TDV1000. Foram 
realizadas dez medições ao longo da superfície lateral de uma amostra de cada processo utilizando um rugosímetro modelo Mitutoyo Surftest SJ-210 Série 178. Foram obtidas imagens em Microscópio Eletrônico de Varredura (MEV) e análises químicas pontuais via Espectroscopia de Energia Dispersiva EDS) da superfície das amostras utilizando um mcroscópio EVO10.

\section{RESULTADOS E DISCUSSÃO}

A Tabela 3 mostra os resultados de densificação (d) das amostras para ambos os processos, antes e após a sinterização.

Tabela 3. Resultado de densificação das amostras

\begin{tabular}{lcccc}
\hline & $\mathbf{d}_{\text {verde }}(\mathbf{\%})$ & $\mathbf{d}_{\text {sinterizado }}(\mathbf{\%})$ & $\boldsymbol{\Delta d} \mathbf{d \% )}$ & Amostragem \\
\hline Convencional & $96,61 \pm 0,44$ & $93,47 \pm 0,51$ & $-3,14 \pm 0,58$ & 4 \\
\hline Plasma & $95,74 \pm 0,36$ & $93,43 \pm 0,34$ & $-2,31 \pm 0,47$ & 6 \\
\hline
\end{tabular}

Pode-se observar que houve uma queda na densificação após a sinterização, para ambos os processos. Este fenômeno, já relatado na literatura [5], [6], [7], trata-se da camada de óxido presente no alumínio, a qual é de difícil redução e prejudica a interdifusão entre as partículas de pó durante a sinterização resultando numa queda na densificação após o processo. No entanto, a análise das variações de densificação revelam que houve menor queda de densidade na sinterização a plasma.

A Figura 2 mostra os valores de microdureza superficial aparente para os processos de sinterização convencional e a plasma. Foram realizadas dez medidas de uma amostra de cada processo com carga de $50 \mathrm{~g}$.

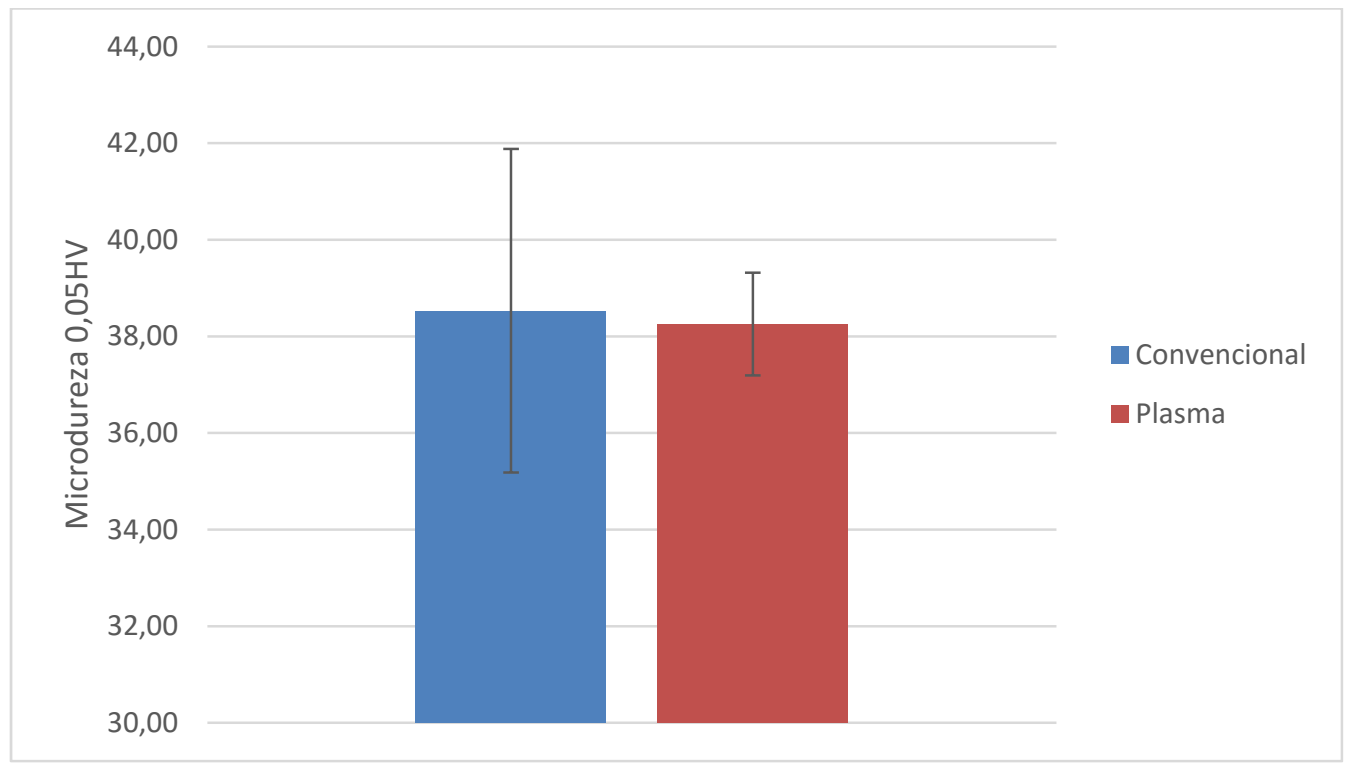

Figura 2. Resultados de microdureza

Pode-se analisar que ambos os processos produziram amostras com microdureza semelhante, sendo dentre eles a sinterização convencional a proporcionar maior variação de dureza. Os resultados encontrados estão condizentes com os valores obtidos para o alumínio puro e com pouco teor de liga [3], [8], [9], [10]. 
Os valores de rugosidade, em termos de Ra e Rz, constam na Tabela 4. Pode-se perceber que a rugosidade obtida em ambos os processos é similar, havendo uma leve redução no processo a plasma com gaiola catódica em relação ao processo convencional.

Tabela 4. Medidas de rugosidade

\begin{tabular}{lcc}
\hline Processo & $\mathbf{R a}(\boldsymbol{\mu m})$ & $\mathbf{R z}(\boldsymbol{\mu m})$ \\
\hline Convencional & $0,84 \pm 0,31$ & $5,15 \pm 1,62$ \\
\hline Plasma & $0,64 \pm 0,16$ & $4,50 \pm 0,79$ \\
\hline
\end{tabular}

As imagens obtidas em MEV da superfície das amostras podem ser observadas nas Figuras 3 e 4 . Foi utilizada a técnica de elétrons retro espelhados, a qual foi capaz de evidenciar os poros da superfície e os contornos entre as partículas de pó, bem como outros elementos presentes.

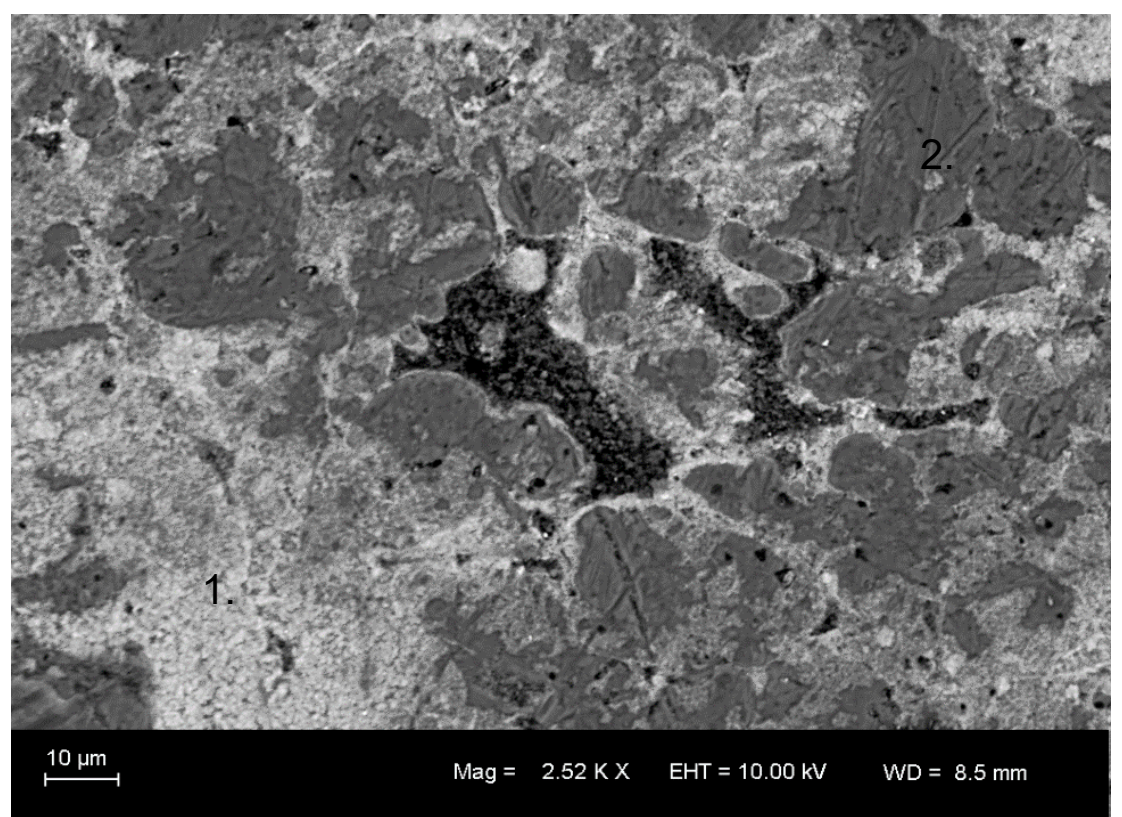

Figura 3. Imagem em MEV da amostra sinterizada pelo método convencional

Através das imagens obtidas com MEV foram escolhidos pontos para as análises via EDS, as quais constam nas Tabelas 5 e 6.

Tabela 5. Análise via EDS referente à Figura 2

\begin{tabular}{ccccccccc}
\hline & \multicolumn{8}{c}{ Composição química } \\
\hline Medição & $\mathbf{C}$ & $\mathbf{O}$ & $\mathbf{M g}$ & $\mathbf{A l}$ & $\mathbf{S i}$ & $\mathbf{C a}$ & $\mathbf{Z n}$ & Total \\
\hline Ponto 1 & 6.59 & 17.15 & 0.10 & 34.84 & 0.08 & 0.29 & 40.95 & 100.00 \\
\hline Ponto 2 & 4.17 & 2.29 & -0.13 & 93.08 & 0.04 & -0.07 & 0.62 & 100.00 \\
\hline
\end{tabular}



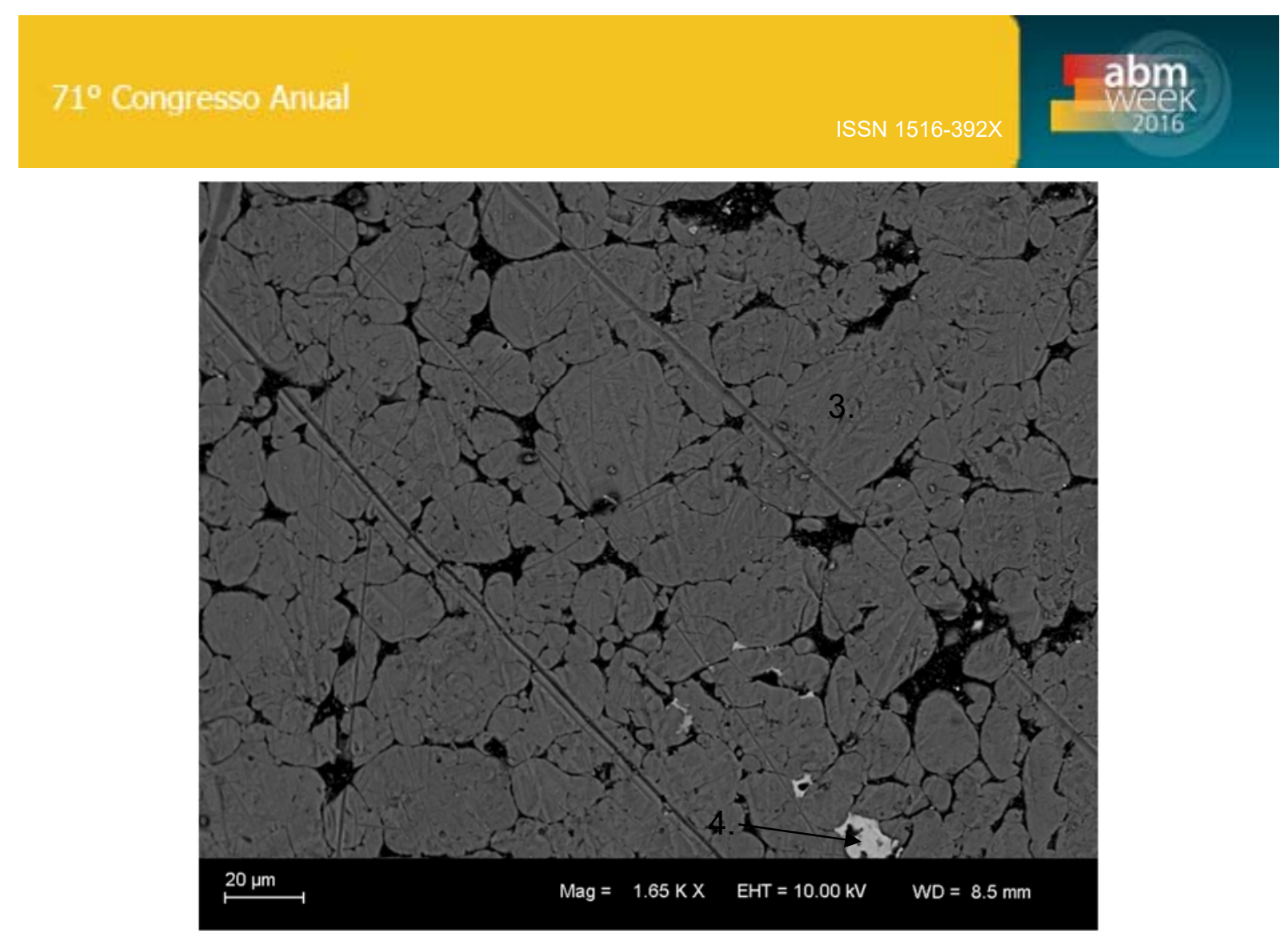

Figura 4. Imagem em MEV da amostra sinterizada via plasma

Tabela 6. Análise via EDS referente à Figura 3

\begin{tabular}{ccccccccccc}
\hline \multicolumn{10}{c}{} & \multicolumn{10}{c}{ Composição química } \\
\hline Medição & $\mathbf{C}$ & $\mathbf{O}$ & $\mathbf{F}$ & $\mathbf{M g}$ & $\mathbf{A l}$ & $\mathbf{S i}$ & $\mathbf{S}$ & $\mathbf{C a}$ & $\mathbf{Z n}$ & Total \\
\hline Ponto 3 & 5.26 & 2.11 & 0.69 & -0.13 & 91.77 & 0.03 & 0.05 & 0.05 & 0.17 & 100.00 \\
\hline Ponto 4 & 19.67 & 20.12 & 0.35 & 0.11 & 12.12 & 0.06 & 0.21 & 0.29 & 47.08 & 100.00 \\
\hline
\end{tabular}

Pode ser observado que os pontos 1 e 4 nas Figuras 2 e 3 evidenciam uma quantidade considerável de zinco na sua composição química, sendo uma forte evidência da presença de estearato de zinco residual após a sinterização.

\section{CONCLUSÃO}

O processo a plasma se mostrou satisfatório, sendo capaz de produzir amostras com boa densificação. As medições de microdureza superficial evidenciaram valores semelhantes entre os processos, sendo a amostra sinterizada via plasma a apresentar menor variação de microdureza.

Através das imagens obtidas em MEV, foi possível constatar a presença de estearato de zinco ainda presente na superfície das amostras após a sinterização, mostrando a necessidade de reavaliar os patamares de tempo/temperatura utilizados para extração do lubrificante, bem como a real necessidade do mesmo. Ainda, houve uma leve redução da rugosidade no processo a plasma em relação ao convencional.

Os resultados obtidos servirão de base para os estudos seguintes, os quais terão como foco a variação de parâmetros de processo e a investigação de um processo combinado de sinterização e nitretação a plasma do alumínio, visando produzir peças com melhores propriedades mecânicas em relação aos métodos convencionais, bem como um processo com maior eficiência energética. 


\section{Agradecimentos}

Ao CNPQ pelo fomento da bolsa de Mestrado, à UFRGS pela infraestrutura e equipamentos utilizados, ao Prof. Dr. Alexandre da Silva Rocha, orientador deste trabalho e aos coautores pelo suporte prestado.

\section{REFERÊNCIAS}

1 Li CX, Bell T, Dong H. A study of Active Screen Plasma Nitriding. Surface Engineering. 2002;18(3):174-181.

2 Klein AN et al. DC Plasma Technology Applied to Powder Metallurgy: An Overview. Plasma Science and Technology. 2013;15(1):70-81.

3 Garcia GS, Gilceu C, Ely LL, Arenhardt SL, Marinho da Silva M, Rocha AS. Estudo da influência da atmosfera na sinterização a plasma do alumínio puro. Seminário de Inovação e Tecnologia do IFSul. 2015;4:324-335.

4 Schubert T et al. P/M Aluminium Structural Parts for Automotive Application. Euro PM: PM Applications. 2004.

5 Schaffer GB. Powder Processed Aluminium Alloys. Materials Forum. 2004;28:65-74.

6 ASM International. Conventional Aluminum Powder Metallurgy Alloys. ASM Handbook. $1998 ; 7$.

7 Lumley R. Fundamentals of aluminium metallurgy. Cambridge: Woodhead Publishing Limited: 2011.

8 Arenhardt SL, Marinho da Silva M, Aurélio dos Santos M, Garcia GS, Martins V, Rocha AS. Estudo do comportamento do Alumínio (Al) com adição de 3\% Cobre (Cu) obtida pela metalurgia do pó convencional. Seminário de Inovação e Tecnologia do IFSul. 2015:4;42-54.

9 Arenhardt SL, Marinho da Silva M, Garcia GS, Martins V, Rocha AS. Estudo do comportamento do alumínio $(\mathrm{Al})$ com adição de !\% cobre $(\mathrm{Cu})$ obtido pela metalurgia do pó convencional. Ciência e Engenharia. 2015;24(1):99-104.

10 Schaffer GB, Hall BJ. The influence of the Atmosphere on the Sintering of Aluminum. Metallurgical and Materials Transactions A. 2002;33:3279-3284. 